\title{
Comparison of Five-Year Survival Rate Between Black and White Children With Acute Lymphoblastic Leukemia
}

\author{
Courtney Bryant ${ }^{1}$, Mackenzie Mayhew ${ }^{1}$, Jorge Fleites ${ }^{1}$, Juan Lozano ${ }^{2}$, John M. Saunders ${ }^{3}$ \\ 1. Pediatric Oncology, Florida International University, Herbert Wertheim College of Medicine, Miami, USA 2. \\ Translational Medicine, Florida International University, Herbert Wertheim College of Medicine, Miami, USA 3. \\ Pediatric Medicine, Florida International University, Herbert Wertheim College of Medicine, Miami, USA
}

Corresponding author: Courtney Bryant, cbrya039@fiu.edu

\section{Abstract}

\section{Introduction}

Despite improvements in the prognosis of acute lymphoblastic leukemia (ALL), it is still the most common childhood cancer. The goal of this study was to investigate if there was a significant difference in the fiveyear survival between Black and White children with ALL, specifically up to the year 2016 which has not been researched.

\section{Methods}

A retrospective cohort study of Black and White children diagnosed with ALL between 1975 and 2016 was carried out using the Surveillance, Epidemiology, and End Results (SEER) Program database. Children aged 0-19 were separated into Black or White, and then survival analysis was used to compare five-year survival. A multivariate cox regression analysis was carried out to determine the association between race and fiveyear survival with ALL.

\section{Results}

Our sample included 17,663 cases consisting of 16,238 White children and 1,425 Black children. White children had a significantly increased five-year mortality survival when compared to Black children. Upon using multivariate cox regression analysis, both unadjusted and adjusted models showed a significantly higher risk of death in Black children when compared to White children.

\section{Conclusions}

Our study found that there is a significant difference in the five-year survival between Black and White children diagnosed with ALL. The difference in survival persists even when controlling for sex, age at diagnosis, year of diagnosis, and histology. Future studies should be carried out to control for more confounders that the SEER database is unable to control for.

Review began $11 / 03 / 2020$ Review ended 11/15/2020 Published 11/30/2020

\section{๑) Copyright 2020}

Bryant et al. This is an open access article distributed under the terms of the Creative Commons Attribution License CC-BY 4.0., which permits unrestricted use, distribution, and reproduction in any medium, provided the original author and source are credited.
Categories: Pediatrics, Oncology

Keywords: race, survival, cancer, acute lymphoblastic leukemia, acute lymphoblastic leukemia (all)

\section{Introduction}

Although there have been major improvements in the prognosis of acute lymphoblastic leukemia (ALL), it is still the most common childhood cancer [1]. ALL can arise through either a T-cell or B-cell lineage [2]. Many different risk factors contribute to this childhood cancer, and although the overall survival rate is now $90 \%$, it is still the most frequent cause of death among this cohort [2]. Factors associated with poor prognosis in ALL include male gender, age at diagnosis (<one year or > 10 years), chromosomal abnormalities, hypodiploidy, T-cell immunophenotype, and peripheral white blood cell count greater than 50000/ $\mu \mathrm{L}[3]$. The clinical trials that use this information to provide risk-adaptive care succeeded in decreasing mortality and toxicity among children with ALL as well as increase their relapse-free and overall survival[1].

Whether race/ethnicity affects the survival rate of ALL and by how much has been the topic of the question with mixed results among different studies and clinical centers [3]. The St. Jude's Research Hospital performed a study in which they used a 30-year study period and found that when looking at the early treatment era, there was a significant difference in the five-year survival rates of Blacks and Whites. When looking at the recent treatment era, however, they found that there was no significant difference [4]. Another study looking at the B-precursor ALL found an excess mortality rate of $42 \%$ in Black children compared with White children after adjusting for other factors such as age, sex, the era of treatment, leukocyte count, and leukemia blast cell ploidy [5]. 
Overall, several studies have looked at the differences in the five-year survival rate of Black and White children with ALL; however, confounding variables such as social determinants of health and access to care are also important factors that many studies have failed to assess. Our study explored the survival rate differences between Black and White children diagnosed with ALL to make steps towards providing equal and unbiased care for every child diagnosed. We also wanted to determine if the gap in survival between races changed over time.

\section{Materials And Methods}

\section{Study design}

This was a retrospective cohort study using the Surveillance, Epidemiology, and End Results (SEER) Program database, a program of the National Cancer Institute (NCI) created in 1973 [6]. It looks at cancer incidence and survival among patients in the United States. Currently, the database includes information on $34.6 \%$ of the US population using population-based cancer registries. The program is updated annually and collects data on patient demographics, primary tumor site, tumor morphology and stage at diagnosis, the first course of treatment, and follow-up for vital status. The mortality date received by the SEER program originates from the National Center for Health Statistics. To ensure that data quality remains high, the NCI staff work with the North American Association of Cancer Registries (NAACR) to help all state registries with their data content and compatibility for acceptable pooling of data and improvement in national estimates. Since we used de-identified data from the SEER database, the study is considered exempt from full review from the institutional review board.

\section{Population and sample}

Our study included children in the age range of 0-19 years old who were diagnosed with ALL between 1975 and 2016. We chose this age range because it includes more cases of ALL in this cohort and there were many previous studies to compare our findings.

\section{Variables}

The independent variable of this study (exposure) was race. Although the SEER database has four categories for race, White, Black, American Indian/Alaskan Native, and Asian or Pacific, for this particular study we only looked at White and Black.

The dependent variable of the study (outcome) is the true five-year survival rate measured by survival analysis.

Confounders accounted for were: sex, age in years at diagnosis (separated into four age groups: 1-4, 5-9, 10 14, 15-19), year of diagnosis between 1975 and 2016, and histology type based on histology codes as per the third edition of International Classification of Diseases for Oncology (ICD-O-3).

\section{Analysis}

Survival analysis was used to assess the two groups' five-year survival rate. Kaplan-Meier survival curves were plotted according to race. Also, both unadjusted and adjusted Cox regression models were used to determine if there were any associations between race and survival. Upon completion, hazard ratios and their corresponding 95\% intervals were calculated allowing for proportional hazard assumptions to be tested graphically.

\section{Results}

\section{Sample characteristics}

After using the SEER database to search for Black and White pediatric patients diagnosed with ALL in the study period, 17,663 cases met our inclusion criteria (Figure 1). Of the cases included, there were 16,238 (91.9\%) White children and 1,425 (8.1\%) Black children. Table 1 summarizes the baseline characteristics of the cohorts. There was no significant difference in sex distribution between both groups $(\mathrm{p}=0.83)$. There was a significant difference in the distribution of cases within the age groups, with slightly more children aged 14 years among Whites than in Blacks (47.7\% vs $41.5 \%$, respectively; $\mathrm{p}<.001)$. There was no significant difference between the two groups of children on the year of diagnosis $(p=0.22)$. B-cell subtype of leukemia made up the majority of the cases in histology for both groups but was significantly more frequent among White than in Black patients (95.1\% vs $89.7 \%$; $<<.001$ ). 


\section{Cureus}

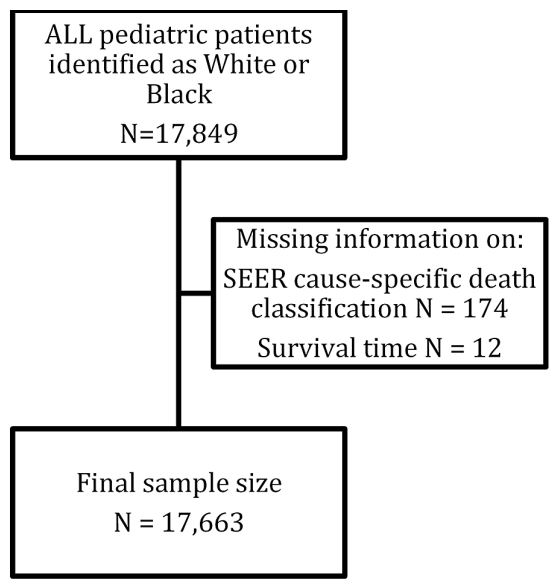

FIGURE 1: Summary of the inclusion and exclusion of cases from the SEER database search 


\section{Cureus}

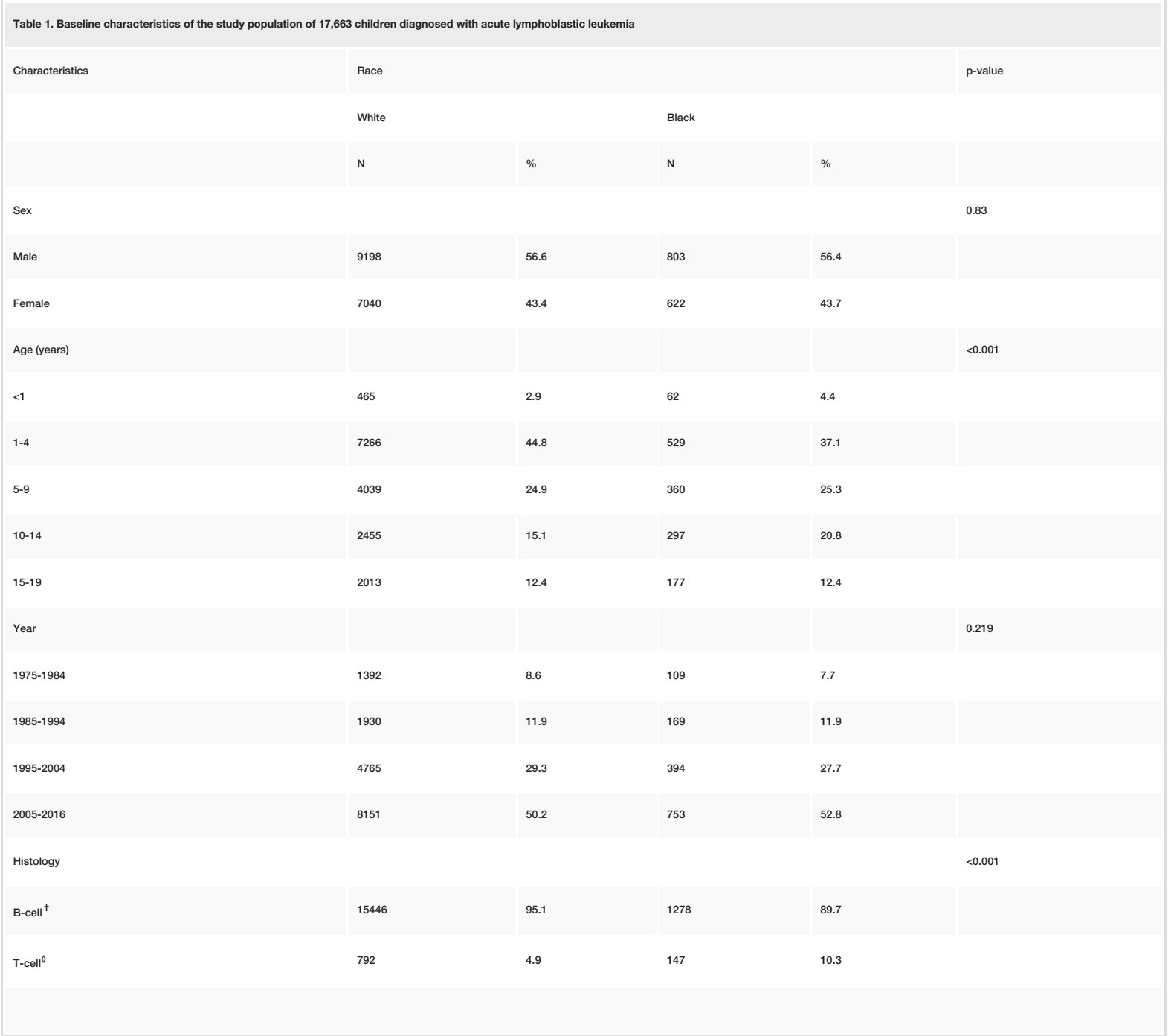

TABLE 1: Baseline characteristics of the study population of 17,663 children diagnosed with acute lymphoblastic leukemia

ICD-O-3 codes included in histology:

†: 9811/3 - B lymphoblastic leukemia/lymphoma, NOS; 9812/3 - Leukemia/lymphoma with t(9;22)(q34;q11.2);BCR-ABL; 9813/3 Leukemia/lymphoma with t(v;11q23);MLL rearranged; 9814/3 - Leukemia/lymphoma with t(12;21)(p13;q22);TEL-AML1(ETV6-RUNX1); 9815/3 - B lymphoblastic leukemia/lymphoma with hyperdiploidy; 9816/3 - Leukemia/lymphoma with hypodiploidy (hypodiploid ALL); 9817/3 - B

lymphoblastic leukemia/lymphoma with $\mathrm{t}(5 ; 14)(\mathrm{q} 31 ; \mathrm{q} 32) ; \mathrm{IL} 3-\mathrm{IGH} ; 9818 / 3$ - Leukemia/lymphoma with $\mathrm{t}(1 ; 19)(\mathrm{q} 23 ; \mathrm{p} 13.3)$; E2A PBX1 (TCF3 PBX1); 9826/3 - Burkitt cell leukemia; 9835/3 - Precursor cell lymphoblastic leukemia, NOS; 9836/3 - Precursor B-cell lymphoblastic leukemia, ৩: 9837/3 -

T lymphoblastic leukemia/lymphoma

\section{Survival analysis}

Overall, White children had a significantly increased five-year survival when compared to Black children $(p<0.001)$. In addition, survival is significantly increased in White children when compared to Black children, as seen in the Kaplan-Meier curve in Figure 2. Furthermore, there were significant differences $(\mathrm{p}<0.001)$ found in the survival according to sex, age, and year of diagnosis (Table 2). Females had an increased survival rate when compared to males. Children between 1-9 years of age had increased survival compared to the other age subgroups. Lastly, both groups of children diagnosed with ALL between 1995 and 2016 had increased survival compared to the children diagnosed between 1975 and 1994. 


\section{Cureus}

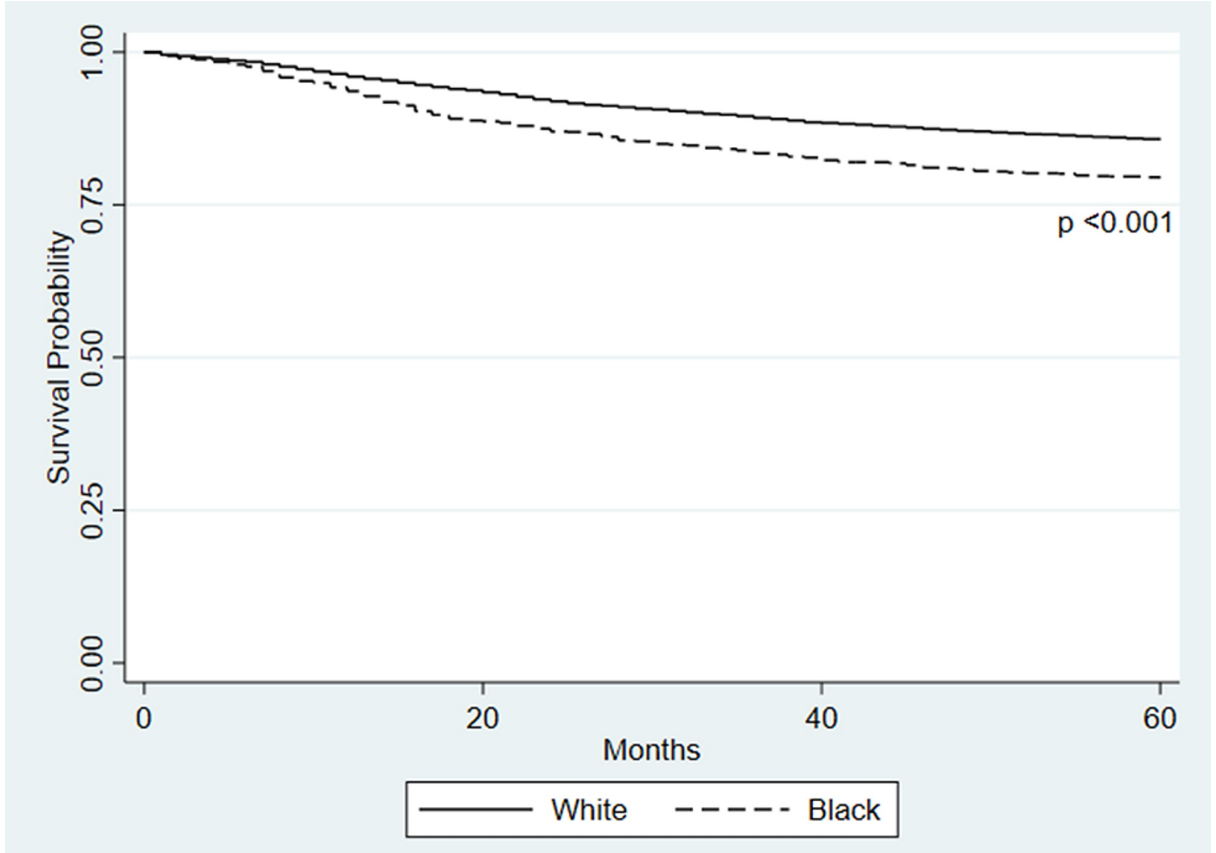

FIGURE 2: Kaplan-Meier survival probability of children according to race 


\section{Cureus}

\begin{tabular}{|c|c|c|c|c|}
\hline Characteristics & Expected & Observed & Log Rank $\mathrm{X}^{2}$ test & p-value \\
\hline Race & & & 41.24 & $<0.001$ \\
\hline White & 2115.5 & 2033 & & \\
\hline Black & 179.5 & 262 & & \\
\hline Sex & & & 40.01 & $<0.001$ \\
\hline Male & 1291.9 & 1442 & & \\
\hline Female & 1003.1 & 853 & & \\
\hline Age (years) & & & 1314.7 & $<0.001$ \\
\hline$<1$ & 49.7 & 198 & & \\
\hline 1-4 & 1065.7 & 561 & & \\
\hline 5-9 & 587.3 & 420 & & \\
\hline 10-14 & 341.2 & 513 & & \\
\hline 15-19 & 251.1 & 603 & & \\
\hline Year & & & 709.3 & $<0.001$ \\
\hline 1975-1984 & 189.2 & 512 & & \\
\hline 1985-1994 & 294.7 & 392 & & \\
\hline 1995-2004 & 754.2 & 687 & & \\
\hline $2005-2016$ & 1057 & 704 & & \\
\hline Histology & & & 1.63 & 0.201 \\
\hline B-cell ${ }^{+}$ & 2187 & 2174 & & \\
\hline T-cell ${ }^{0}$ & 108.1 & 121 & & \\
\hline
\end{tabular}

TABLE 2: Survival probabilities among the study population of 17,663 children diagnosed with acute Iymphoblastic leukemia

ICD-O-3 codes included in histology:

†: 9811/3 - B lymphoblastic leukemia/lymphoma, NOS; 9812/3 - Leukemia/lymphoma with t(9;22)(q34;q11.2);BCR-ABL; 9813/3 Leukemia/lymphoma with t(v;11q23);MLL rearranged; 9814/3 - Leukemia/lymphoma with t(12;21)(p13;q22);TEL-AML1(ETV6-RUNX1); 9815/3 - B lymphoblastic leukemia/lymphoma with hyperdiploidy; 9816/3 - Leukemia/lymphoma with hypodiploidy (hypodiploid ALL); 9817/3 - B lymphoblastic leukemia/lymphoma with $\mathrm{t}(5 ; 14)(\mathrm{q} 31 ; \mathrm{q} 32) ; \mathrm{IL3}-\mathrm{IGH} ; 9818 / 3$ - Leukemia/lymphoma with t(1;19)(q23;p13.3); E2A PBX1 (TCF3 PBX1); 9826/3 - Burkitt cell leukemia; 9835/3 - Precursor cell lymphoblastic leukemia, NOS; 9836/3 - Precursor B-cell lymphoblastic leukemia, ৩: 9837/3 T lymphoblastic leukemia/lymphoma

\section{Multivariate cox regression analysis}

To determine the potential confounders included in the Cox regression, collinearity between each was tested. No variables demonstrated collinearity, thus, sex, age at diagnosis, year of diagnosis, and histology were all included in the analysis.

Both unadjusted and adjusted models showed a significantly higher risk of death in Black children as compared to White children (Table 3). The unadjusted risk of death was $52 \%$ greater in Black children (hazard ratio (HR) 1.52, $95 \%$ CI 1.34 to 1.73 ; $\mathrm{P}<0.001$ ). When adjusting for sex, age at diagnosis, year of diagnosis, and histology, Black children had a $45 \%$ higher hazard of death than did White children (adjusted HR $1.45,95 \%$ CI 1.28 to 1.66 ; P0<.001). While the risk of death decreased when adjusting for confounders, Black children still have a significantly greater risk of death. The female children had a lower risk of death than male children in both unadjusted and adjusted models. All age groups had a significantly lower risk of 


\section{Cureus}

death than children in the $<1$ year age group in both unadjusted and adjusted models. There was a significantly lower risk of death in the time period of 2005-2016 than in the decades before, both unadjusted and adjusted models. When unadjusted, histology was not significantly associated with differences in survival $(\mathrm{P}=.202)$, but when adjusted, it showed a significantly higher risk of death in the T-cell lineage (aHR $1.22,95 \%$ CI 1.01 to $1.48 ; \mathrm{P}<0.05)$ than the B-cell lineage.

The findings of the analysis looking at the gap in survival between the two races along the four decades of observation are depicted in Table 4. Despite some trends towards improvement, survival was significantly lower for Black children along all four periods of observation. Adjusted hazard ratios ranged from 1.9 (95\% CI 1.4-2.5, $\mathrm{p}<0.001)$ and $1.3(95 \%$ CI 1.0-1.6, $\mathrm{p}=0.03)$, showing that the inequalities persist throughout time.

\begin{tabular}{|c|c|c|c|c|}
\hline \multirow[t]{2}{*}{ Characteristics } & Unadjusted & & Adjusted & \\
\hline & $\mathrm{HR}(95 \% \mathrm{Cl})$ & p-value & HR (95\% Cl) & p-value \\
\hline \multicolumn{5}{|l|}{ Race } \\
\hline White & reference & & reference & \\
\hline Black & $1.52(1.34-1.73)$ & $<0.001$ & $1.45(1.28-1.66)$ & $<0.001$ \\
\hline \multicolumn{5}{|l|}{ Sex } \\
\hline Male & reference & & reference & \\
\hline Female & $0.76(0.70-0.83)$ & $<0.001$ & $0.82(0.75-0.89)$ & $<0.001$ \\
\hline \multicolumn{5}{|l|}{ Age (years) } \\
\hline$<1$ & reference & & reference & \\
\hline 1-4 & $0.13(0.11-0.15)$ & $<0.001$ & $0.13(0.11-0.15)$ & $<0.001$ \\
\hline 5-9 & 0.18 (0.15-0.21) & $<0.001$ & $0.18(0.15-0.21)$ & $<0.001$ \\
\hline 10-14 & 0.38 (0.32--0.44) & $<0.001$ & $0.37(0.31-0.44)$ & $<0.001$ \\
\hline 15-19 & $0.60(0.51-0.71)$ & $<0.001$ & $0.61(0.52-0.71)$ & $<0.001$ \\
\hline \multicolumn{5}{|l|}{ Year } \\
\hline 1975-1984 & $4.07(3.63-4.56)$ & $<0.001$ & $4.43(3.94-4.98)$ & $<0.001$ \\
\hline 1985-1994 & $2.00(1.77-2.26)$ & $<0.001$ & 2.24 (1.98-2.54) & $<0.001$ \\
\hline 1995-2004 & $1.37(1.23-1.52)$ & $<0.001$ & $1.47(1.32-1.63)$ & $<0.001$ \\
\hline 2005-2016 & reference & & reference & \\
\hline \multicolumn{5}{|l|}{ Histology } \\
\hline B-cell ${ }^{+}$ & reference & & reference & \\
\hline T-celli ${ }^{0}$ & $1.13(0.94-1.35)$ & 0.202 & $1.22(1.01-1.48)$ & $<0.05$ \\
\hline
\end{tabular}

TABLE 3: Multivariate Cox regression with unadjusted and adjusted associations between acute lymphoblastic leukemia and race, sex, age at diagnosis, year of diagnosis, and histology

Abbreviations: $\mathrm{Cl}$, confidence interval; HR, hazard ratio. ICD-O-3 codes included in histology:

†: 9811/3 - B lymphoblastic leukemia/lymphoma, NOS; 9812/3 - Leukemia/lymphoma with t(9;22)(q34;q11.2);BCR-ABL; 9813/3 Leukemia/lymphoma with t(v;11q23);MLL rearranged; 9814/3 - Leukemia/lymphoma with t(12;21)(p13;q22);TEL-AML1(ETV6-RUNX1); 9815/3 - B lymphoblastic leukemia/lymphoma with hyperdiploidy; 9816/3 - Leukemia/lymphoma with hypodiploidy (hypodiploid ALL); 9817/3 - B lymphoblastic leukemia/lymphoma with $\mathrm{t}(5 ; 14)(\mathrm{q} 31 ; \mathrm{q} 32) ; \mathrm{IL3}-\mathrm{IGH} ; 9818 / 3$ - Leukemia/lymphoma with t(1;19)(q23;p13.3); E2A PBX1 (TCF3 PBX1); 9826/3 - Burkitt cell leukemia; 9835/3 - Precursor cell lymphoblastic leukemia, NOS; 9836/3 - Precursor B-cell lymphoblastic leukemia, ৩: 9837/3 T lymphoblastic leukemia/lymphoma 


\section{Cureus}

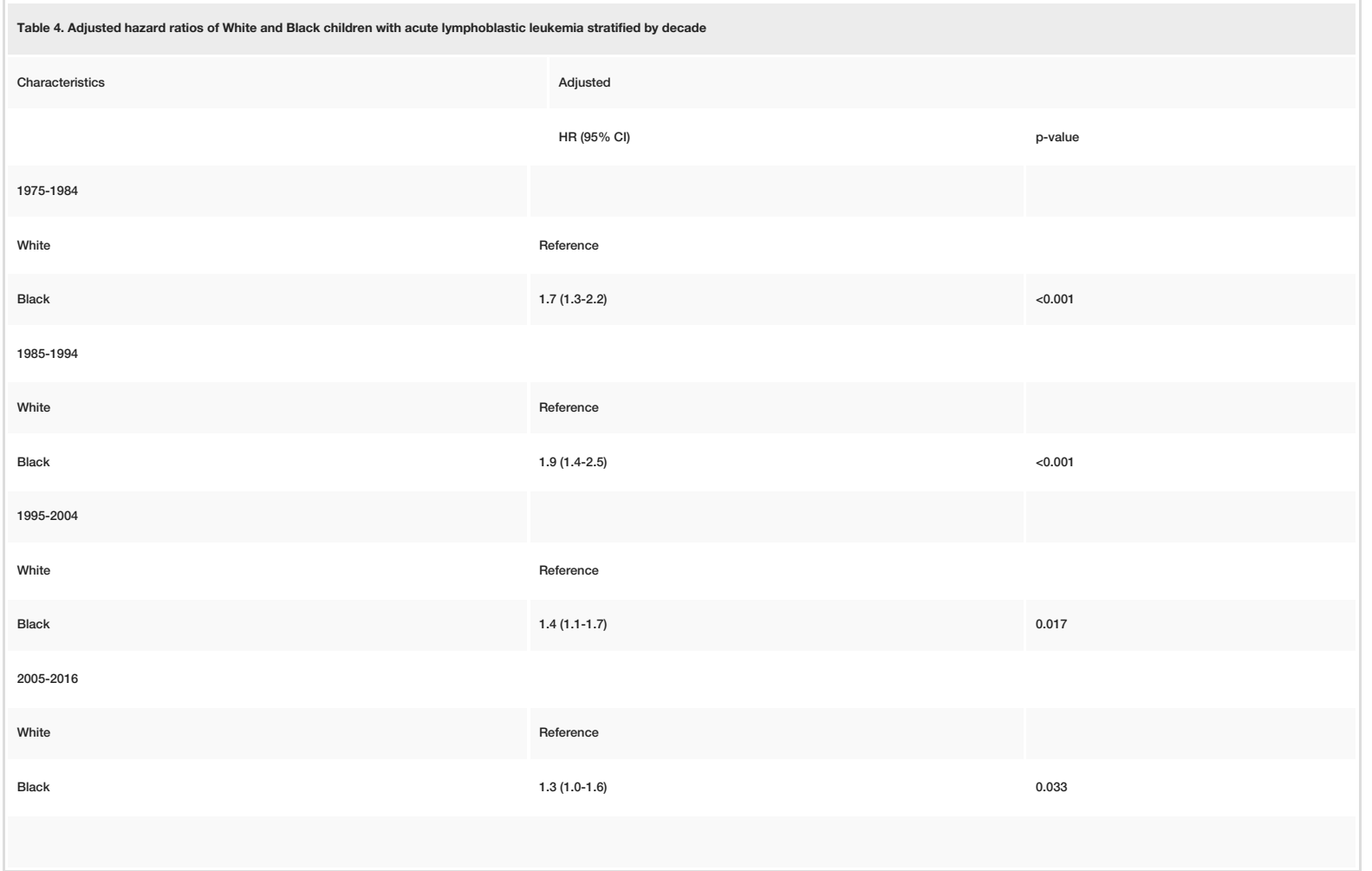

TABLE 4: Adjusted hazard ratios of White and Black children with acute lymphoblastic leukemia stratified by decade

Abbreviations: $\mathrm{Cl}$, confidence interval; $\mathrm{HR}$, hazard ratio

\section{Discussion}

ALL continues to be the most common childhood cancer [1]. Treatment for ALL has made many advances throughout the years leading to a survival rate of over $90 \%$ in children. Many past studies have contributed to the high survival rate through research on risk and prognostic factors. Our primary goal of this study was to compare the survival of White and Black children to expose any inequalities that still exist and move closer to bridging the gap in survival between the two races.

This study looked at White and Black children ages 0-19 years old who were diagnosed with ALL. We used the SEER database looking at all relevant diagnoses from the years 1975-2016, excluding only those with insufficient data about survival. After controlling for confounders we found a statistically significant difference in the survival rates between the two races. In addition, we also found that among both races females had an increased survival rate. The age group 1-4 years also had the most cases of ALL. Our findings were congruent with other studies in the past which found that White children have a higher five-year survival rate compared to Black children [1,3-5,7-12]. Between 1995-2016 we found that there was the highest five-year survival rate among both races compared to other years possibly due to the advances in treatment and research on this type of cancer.

Confounders for this study were chosen based on previous studies [1,3-5,7-12]. However, none of these studies looked at data past the year 2009. Since our study includes patients through 2016, it allows for more recent, modern advances and survival data to be taken into account. Due to the fact that we used the SEER database, there were some confounders we were not able to assess because the database did not have these variables. These include socioeconomic status, access to healthcare, genetic risk factors, other comorbidities, disease-specific treatment and relapse data. One study performed by Pui et al. looked at two different populations, one from the SEER database and another from the St. Jude's Research Hospital [11]. This study was unique in that St. Jude's Research Hospital gives comprehensive care to every patient regardless of their ability to pay. Although this study only looked at data up to 2007, they found that when access to care was controlled for there were no significant differences in survival rates among Black and White children. Although our study could not control for this confounder, we did find that the survival rate between the two races continues to narrow as advances in treatment continue to be made. Additionally, we found that the difference in survival begins between about 12 to 18 months. Given the findings of the Pui et al. study [11] and that the typical treatment of ALL has three phases [13] - remission induction, 
consolidation/intensification, maintenance - with the first and second phases lasting between six and eight months [13], we can reasonably postulate that access to care and other socioeconomic issues contribute to the difference in five-year survival between Black and White children.

The strengths of this study include the fact that it was a retrospective study allowing it to be performed quickly and inexpensively. We used a de-identified database giving few ethical issues and allowing us to bypass IRB. However, there were some limitations. For example, the inability to control for access to care and take into account other confounders limited our study. In addition, due to the type of cancer we researched, the grade could not be accounted for either. Instead, histology type was used to parallel the reasoning behind this variable. Therefore, our study shows that, despite some improvement in the last four decades, inequalities in survival have persisted for a long time.

\section{Conclusions}

In conclusion, our study supported that there continues to be a significant difference in the five-year survival rate between Black and White children diagnosed with ALL. Additionally, the rate at which children die is significantly higher in Black children. Sex, age, and year of diagnosis are important confounding variables, but even when controlled for, did not eliminate the difference in survival rates. Future research studies should be done to control for more confounders that were unable to be controlled for using the SEER database. Therefore, studies should also attempt to build off of St. Jude's Research Hospital to look at the survival rates of different pediatric populations while specifically controlling for access to care.

\section{Additional Information \\ Disclosures}

Human subjects: Consent was obtained by all participants in this study. Animal subjects: All authors have confirmed that this study did not involve animal subjects or tissue. Conflicts of interest: In compliance with the ICMJE uniform disclosure form, all authors declare the following: Payment/services info: All authors have declared that no financial support was received from any organization for the submitted work. Financial relationships: All authors have declared that they have no financial relationships at present or within the previous three years with any organizations that might have an interest in the submitted work. Other relationships: All authors have declared that there are no other relationships or activities that could appear to have influenced the submitted work.

\section{References}

1. Tai EW, Ward KC, Bonaventure A, Siegel DA, Coleman MP: Survival among children diagnosed with acute lymphoblastic leukemia in the United States, by race and age, 2001 to 2009: Findings from the CONCORD-2 study. Cancer. 2017;123, 24:5178-5189. 10.1002/cncr.30899

2. Hunger SP, Mullighan CG: Acute lymphoblastic leukemia in children . New Eng J Med. 2015, 373:1541-1552. 10.1056/NEJMra1400972

3. Kadan-Lottick NS, Ness KK, Bhatia S, Gurney JG: Survival variability by race and ethnicity in childhood acute lymphoblastic leukemia. JAMA. 2003, 290:2008-2014. 10.1001/jama.290.15.2008

4. Pui CH, Boyett JM, Hancock ML, Pratt CB, Meyer WH, Crist WM: Outcome of treatment for childhood cancer in black as compared with white children. The St Jude Children's Research Hospital experience, 1962 through 1992. JAMA. 1995, 273:633-637. 10.1001/jama.1995.03520320043039

5. Pollock BH, DeBaun PB, Camitta M, et al.: Racial differences in the survival of childhood B-precursor acute lymphoblastic leukemia: a Pediatric Oncology Group Study. J Clin Oncol. 2000, 18:813-23. 10.1200/JCO.2000.18.4.813

6. National Cancer Institute. Overview of the SEER Program . (2020). Accessed: May 12, 2020: https://seer.cancer.gov/about/overview.html.

7. Zhao Y, Wang Y, Ma S: Racial differences in four leukemia subtypes: comprehensive descriptive epidemiology. Sci Rep. 2018, 8:548. 10.1038/s41598-017-19081-4

8. Acharya S, Hsieh S, Shinohara ET, DeWees T, Frangoul H, Perkins SM: Effects of race/ethnicity and socioeconomic status on outcome in childhood acute lymphoblastic leukemia. J Pediatr Hematol Oncol. 2016, 38:350-354. 10.1097/MPH.0000000000000591

9. Kahn JM, Keegan TH, Tao L, Abrahão R, Bleyer A, Viny AD: Racial disparities in the survival of American children, adolescents, and young adults with acute lymphoblastic leukemia, acute myelogenous leukemia, and Hodgkin lymphoma. Cancer. 2016, 122:2723-2730. 10.1002/cncr.30089

10. Hunger SP, Lu X, Devidas M, et al.: Improved survival for children and adolescents with acute lymphoblastic leukemia between 1990 and 2005: a report from the children's oncology group. J Clin Oncol. 2012, 30:16631669. 10.1200/JCO.2011.37.8018

11. Pui CH, Pei D, Pappo AS, et al.: Treatment outcomes in black and white children with cancer: results from the SEER database and St Jude Children's Research Hospital, 1992 through 2007. J Clin Oncol. 2012, 30:20052012. 10.1200/JCO.2011.40.8617

12. Mcneil DE, Coté TR, Clegg L, Mauer A: SEER update of incidence and trends in pediatric malignancies: Acute lymphoblastic leukemia. Med Pediatr Oncol. 2002, 39:554-557. 10.1002/mpo.10161

13. Children's Oncology Group. Treating acute lymphoblastic leukemia . (2011). Accessed: Nov 11, 2020: https://www.childrensoncologygroup.org/index.php/in-treatment-for-acute-lymphoblastic-leukemia . 